\title{
Le lait et les produits laitiers en République Populaire de Mongolie
}

\author{
par \\ J. P. ACCOLAS \\ Laboratoire de Technologie Laitière, I.N.R.A. \\ 78350 Jouy-en-Josas \\ J. P. DEFFONTAINES \\ Service d'Expérimentation et d'Information, I.N.R.A. \\ 78000 Versailles \\ et Françoise AUBIN \\ Centre National de la Recherche Scientifique, Paris
}

Les informations ici présentées sont extraites de deux articles* composés à partir de données bibliographiques et d'observations personnelles recueillies par les auteurs au cours d'une mission d'étude (J.P.A. et J.P.D., en septembre 1974) et de plusieurs séjours prolongés en Mongolie (F.A., entre 1966 et 1973).

\footnotetext{
* Accolas (J. P.), Deffontaines (J. P.) et Aubin (F.) (1975). - Les activités rurales en République Populaire de Mongolie. I. Agriculture et élevage. II. Les produits laitiers. Etudes Mongoles, Cahier n ${ }^{\circ}$ 6, 7-98. (Publiées sous forme de cahiers périodiques, Etudes Mongoles sont préparées par le Centre d'Etudes Mongoles, Laboratoire d'Ethnologie et de Sociologie Comparative, Université de Paris X 92001 Nanterre).

La transcription des termes mongols a été assurée à l'aide d'un système simplifié de translittération de l'alphabet mongol : $u$ rend le son ou français, $\ddot{u}$ le son $u$, $\ddot{o}$ le son $\alpha, z$ le son $d z$ et les voyelles longues sont indiquées par les voyelles doubles aa, ee, oo, öö, uu, ӥï ou par $i j$ pour le $i$; mais on a employé $t s$ au lieu du $c$ utilisé dans un système de translittération scientifique, $\ddot{i}$ pour rendre la yodisation (j), $k h$ au lieu de $x$ et $s h$ pour rendre le son $c h$.
} 


\section{INTRODUCTION}

La Mongolie, située au centre de l'Asie entre la Sibérie et la Chine, est un pays de montagnes et de hauts plateaux soumis à un climat continental rigoureux à caractère montagnard sec. Son territoire, $1500000 \mathrm{~km}^{2}$, est constitué, pour 85 p. 100, de pâturages steppiques de nature diverse, ce qui explique sa vocation pastorale séculaire. La densité de population est faible, moins d'un habitant par $\mathrm{km}^{2}$ en moyenne, en 1970. Peu après avoir proclamé son indépendance, le pays est devenu, en 1924, la République Populaire de Mongolie (R.P.M.). Entrée en 1964 dans le Comecon, la R.P.M., bénéficiant de l'aide de l'Union Soviétique et des autres pays socialistes, se transforme actuellement en une sociéte agro-industrielle structurée, sédentarisée et urbanisée. Toutefois, l'élevage demeure l'une des principales activités et l'une des ressources essentielles du pays. Le cheptel mongol comptait, en 1974, près de 24 millions d'animaux qui se répartissaient de la façon suivante (en millions) : ovins, 14 ; caprins, 4,5 ; bovins, 2,3 (y compris 500000 yaks et hybrides) ; chevaux, 2,2 ; chameaux, 0,6, auxquels s'ajoutent un petit élevage de rennes localisé dans la partie nord-ouest du pays et un élevage porcin encore peu développé.

Les peuples nomades d'Asie Centrale ont cherché, depuis les temps reculés où ils ont maîtrisé les techniques de l'élevage, à tirer le meilleur parti possible du lait de leurs différents animaux domestiques, afin de satisfaire, à la fois, leurs besoins alimentaires et leurs goûts. Ils furent, vraisemblablement, parmi les premiers à développer les laits fermentés. Une de leurs préoccupations majeures, en raison du caractère saisonnier de la production laitière, a été aussi de préparer des produits laitiers de report, se conservant bien, qui, secs ou congelés, leur permettaient, conjointement avec la viande séchée, d'assurer leur subsistance pendant le long et rigoureux hiver d'Asie Centrale.

\section{L'ECONOMIE LAITIERE MODERNE}

Dans ce pays en pleine mutation, où l'urbanisation progresse rapidement, l'approvisionnement en lait et en produits laitiers des agglomérations urbaines se pose avec acuité. Pour cette raison, l'Institut d'Elevage de la R.P.M. a reçu mission, à partir de 1965, de moderniser la préparation des produits laitiers, qui présente encore un caractère très artisanal sinon familial. De petites entreprises industrielles pilotes, traitant 8 à 100001 de lait par jour pendant les 5 à 6 mois de production laitière, ont ainsi été créées en divers points du pays, pour fabriquer du beurre et des fromages mongols. 
L'expérience déjà acquise dans ce domaine, du double point de vue technique et économique, a été jugée suffisamment positive pour poursuivre l'implantation de tels ateliers de transformation dans les zones laitières.

La capitale, Ulan-Bator, dispose, depuis une quinzaine d'années, d'une centrale laitière qui a traité, en 1974, 900001 de lait par jour en période de pointe et 200001 en hiver. Le maximum, qui doit être porté à 1500001 dans un avenir proche, sera encore notablement insuffisant pour faire face aux besoins d'une population citadine de plus de 300000 habitants, grande consommatrice de lait. Le lait est collecté, dans un rayon de $200 \mathrm{~km}$ autour de la ville, dans les coopératives d'éleveurs et dans les fermes d'Etat. Après pasteurisation, il est livré en vrac, par camions-citernes, aux différents points de vente au détail d'Ulan-Bator. La centrale laitière prépare aussi, selon ses disponibilités en lait, du beurre, de la crème acidifiée (analogue au smetana russe), du tarag (yoghourt), des glaces et divers fromages secs traditionnels (aaruul).

Il existe aussi, à Ulan-Bator, un petit atelier qui traite 40001 de lait par jour tout au long de l'année, pour préparer des biberons de lait et des aliments infantiles destinés à l'alimentation d'environ 6000 nourrissons et bébés, par l'intermédiaire d'organismes divers (hôpitaux, maternités, centres de distribution de quartier).

\section{TECHNOLOGIE LAITIERE MONGOLE}

Les diverses utilisations traditionnelles du lait en Mongolie témoignent d'une certaine unité, en dépit de particularités propres à chaque région ou à chaque ethnie. Par exemple, dans la zone montagneuse de Khangaï, ce sont les laits de vache et de yak qui prédominent, alors que dans la zone semi-désertique des Gobi, ce sont les laits de chèvre, de brebis et de chamelle. Un peu partout, on trait les juments et très localement, c'est-à-dire dans la partie nord-ouest du pays, les rennes.

Le lait est toujours consommé après un chauffage atteignant presque l'ébullition et la boisson nationale, qui accompagne traditionnellement les repas et qui est servie en toutes occasions, est le thé mongol (süüteï tsaï), c'est-à-dire du lait bouilli additionné de thé, émietté d'une brique de thé compact, d'un peu de sel et fréquemment enrichi avec du beurre fondu et du fromage sec mongols.

\section{Conservation du lait}

Les Mongols utilisent divers procédés artisanaux pour conserver, pendant l'hiver, le lait produit en fin de saison. Mentionnons la congé- 
lation, facile à réaliser en raison des très basses températures hivernales ; la concentration par ébullition prolongée et la conservation au froid du lait concentré; le séchage en couches minces du lait préalablement concentré, le lait sec obtenu, réduit en poudre, étant ensuite conservé à l'abri de l'humidité.

\section{Séparation des matières grasses}

a) La crème (tsötsgij) est obtenue par écrémage centrifuge du lait et transformée ensuite en beurre, par barattage. Cette technique n'offre aucune particularité et elle est d'introduction récente en Mongolie. Elle est utilisée par les petits ateliers industriels et par de nombreuses coopératives d'éleveurs pour fabriquer un beurre " industriel » de type occidental, le tsötsgijn tos.

b) Deux procédés traditionnels sont encore couramment employés en Mongolie pour extraire la matière grasse du lait.

Premier procédé. Il s'agit là du procédé séculaire des nomades de la steppe, qui comporte deux étapes essentielles : la préparation de l’öröm à partir du lait et l'extraction de la matière grasse de l'öröm par fusion, pour obtenir le beurre fondu ou shar tos (litt. beurre jaune).

Préparation de l'öröm : du lait frais (de vache, de yak ou de brebis) est porté à l'ébullition et constamment agité à l'aide d'une louche, pour favoriser la formation de mousse. Le lait est ensuite transvasé dans des récipients de diamètre suffisant et laissé refroidir au repos. Plusieurs réchauffages périodiques à $60^{\circ} \mathrm{C}-70^{\circ} \mathrm{C}$ peuvent être pratiqués pour activer la montée de la matière grasse. Après plusieurs heures, en général le lendemain de la fabrication, il s'est formé, à la surface du lait, une " peau » épaisse $(1,5$ à $2 \mathrm{~cm})$ et dure, riche en matière grasse. C'est l'öröm. Cette peau est généralement prélevée d'un seul bloc et pliée en deux, la couche la plus molle précédemment au contact du lait étant à l'intérieur.

L'öröm ainsi préparé est un mets apprécié des Mongols qui le consomment frais ou séché, éventuellement additionné de produits très divers (riz, mil, pommes de terre, raisins secs, fromage séché réduit en poudre, sucre, etc.).

Préparation du beurre fondu (shar tos) : l'öröm est stocké, sous le nom de tü̈̈khij öröm ou de tü̈̈khij tos, dans un récipient réservé à cet usage, au fur et à mesure de sa préparation quotidienne, pendant plusieurs semaines, voire tout l'été. Soumis à diverses fermentations microbiennes qui modifient sensiblement, au cours de la conservation, ses qualités organoleptiques initiales, l'öröm ainsi fermenté est souvent dénommé zöökhij.

$\mathrm{Au}$ terme de la conservation, la matière grasse est extraite du zöökhij par un chauffage prolongé ( 2 h environ), en agitant constam- 
ment pour hâter l'évaporation de l'eau. On clarifie la matière grasse à l'aide de farine : il se forme alors une masse spongieuse rassemblant les impuretés, qui est recueillie à la louche.

Le beurre fondu, de couleur jaune translucide et de consistance onctueuse est conservé, souvent pendant de longues périodes (plusieurs mois, parfois plusieurs années) dans des estomacs ou des boyaux de chèvre ou de mouton préalablement lavés et séchés au soleil. Le beurre fondu est utilisé dans de nombreuses préparations culinaires. Il est aussi très fréquemment ajouté, en petites quantités, au thé mongol.

Les résidus de fusion, une fois pressés à chaud pour en extraire le maximum de matière grasse et additionnés de farine, de sucre, etc., constituent le tsöv. Conservé de manière analogue au beurre fondu, le tsöv est généralement présenté, à table, en mélange avec les autres produits laitiers traditionnels, sous forme de petits rectangles de couleur brune et de consistance molle.

Deuxième procédé. Dans l'ouest et dans le sud de la Mongolie, le beurre est obtenu par barattage direct du lait (en général du lait de brebis ou de chamelle) dans des barattes à main, après que ce lait ait subi, pendant une courte période de conservation, une acidification naturelle dans des outres en peau ou des seaux en bois réservés à cet usage, lesquels apportent vraisemblablement la flore acidifiante nécessaire. Une fois les grains de beurre formés et rassemblés à la surface du lait, on les recueille avec une écumoire.

D'une façon similaire, les Mongols recueillent aussi le beurre qui se forme au cours de la préparation des laits fermentés acidoalcooliques, en raison de l'agitation très fréquente du lait qui est pratiquée au cours de la fermentation.

\section{Laits fermentés}

Les peuples nomades d'Asie Centrale furent vraisemblablement parmi les premiers à développer les laits fermentés. Ceux-ci relèvent d'une antique tradition et témoignent d'un judicieux esprit d'observation. Tirant parti des «bonnes » fermentations acides et alcooliques que subit naturellement le lait, ces peuples turco-mongols ont en effet préparé, depuis des siècles, des laits fermentés bien adaptés à leur mode de vie et à leurs goûts. Bien entendu, dans la pratique artisanale, il est souvent difficile d'établir une distinction tranchée entre les divers produits, car il arrive très fréquemment qu'un lait fermenté acide, contenant un nombre élevé de levures, subisse un début de fermentation alcoolique. Ces réserves étant faites, on peut distinguer trois types de laits fermentés : 


\section{Tarag}

Etroitement apparenté au yoghourt, le tarag est préparé avec du lait de vache, de yak, de brebis ou de chèvre. Le bon tarag présente une consistance épaisse, dense et homogène, une cassure lisse sans trace de fermentation gazeuse, un goût frais et aigrelet.

On utilise du lait entier qui, après avoir été chauffé à $80^{\circ} \mathrm{C}$ $90^{\circ} \mathrm{C}$, est laissé refroidir à une température voisine de $45^{\circ} \mathrm{C}$, ou, plus fréquemment, du lait sur lequel on a prélevé l'öröm et qu'on réchauffe à $45^{\circ} \mathrm{C}$. On ajoute 5 p. 100 à 20 p. 100 de levain, on transvase le lait ensemencé dans le récipient réservé à la préparation du tarag et on incube au chaud, en couvrant le récipient de tissus isolants pour éviter une chute trop rapide de la température. La coagulation intervient après $2 \mathrm{~h}$ à $4 \mathrm{~h}$ d'incubation.

Les Mongols attachent, à juste titre, une grande importance au levain, le targijn khöröngö. Pendant la saison de production laitière, du printemps à l'automne, le levain est constitué par du tarag de la meilleure qualité, régulièrement renouvelé, qui s'égoutte en permanence dans un petit sac suspendu près de l'outre à koumis, à gauche de la porte d'entrée de la yourte. En fin de saison, à l'approche de l'hiver, on prépare, à partir du tarag, un caillé analogue à l'aarts (cf. ci-dessous), qui est découpé en petits morceaux, congelé ou séché et conservé dans un endroit froid et sec, jusqu'à la reprise de la production laitière, au printemps suivant : c'est le targijn aaruul. Pour réactiver le ferment, on le remet en suspension dans du lait frais bouilli et on incube le mélange. Les résultats sont assez aléatoires, en dépit du soin apporté à la préparation.

\section{Ä̈rag}

Il s'agit là d'un lait fermenté acido-alcoolique presque exclusivement préparé avec du lait de jument, plus connu à l'étranger sous la dénomination de koumis, qui est d'origine turque.

Le lait cru fraîchement trait, c'est-à-dire en moyenne cinq à six fois par jour, est aussitôt versé dans une outre en peau ou dans un récipient en bois, rituellement placé à gauche de l'entrée de la yourte, du côté réservé aux activités masculines. Pendant la saison de production, le récipient contient toujours du lait de jument des jours précédents, en cours de fermentation. L'addition quotidienne de lait frais, qui compense la consommation, permet d'entretenir un processus semi-continu de fermentation, à $\mathrm{pH}$ relativement bas et à une température voisine de la température ambiante. Une agitation très fréquente du contenu du récipient, à l'aide d'un batteur à main, est indispensable pour obtenir une bonne fermentation alcoolique.

L'aïrag est un lait fermenté particulièrement prisé des Mongols, en raison de ses qualités organoleptiques spécifiques. Il se présente en effet comme une boisson légère, piquante et rafraîchissante, dont 
la teneur en alcool peut atteindre 2,5 p. 100 à 3 p. 100 . Relativement pauvre en caséine, le lait de jument acide coagule très finement et le coagulum, aisément remis en suspension, est imperceptible à la dégustation.

On attribue à l'aïrag de multiples propriétés diététiques et thérapeutiques et on l'emploie en Mongolie, comme le koumis en Union Soviétique, dans les établissements de cure où sont soignées des affections variées, en particulier la tuberculose.

On l'utilise aussi pour préparer de l'alcool de lait et des aaruul (cf. ci-dessous).

\section{Undaa, Umdaa, Khoormog}

Ces dénominations regroupent des laits fermentés acido-alcooliques préparés avec les laits des espèces laitières autres que la jument. Leur préparation est analogue à celle de l'aïrag, mais le produit obtenu présente une consistance épaisse et le coagulum, qui se sépare du lactosérum, est difficilement remis en suspension. Pour cette raison, ces laits fermentés sont beaucoup moins appréciés que l'aïrag par les Mongols, et ils sont surtout utilisés pour préparer de l'alcool de lait par distillation, de l'aarts et des aaruul séchés.

\section{Caillés et produits laitiers secs}

En Mongolie, la préparation du caillé et des produits laitiers secs qui en dérivent, met en œuvre traditionnellement une combinaison de l'acidification et du chauffage du lait. On utilise aussi le tarag et les résidus de distillation des laits fermentés acido-alcooliques. On notera que les Mongols n'emploient pas traditionnellement la présure pour obtenir la coagulation du lait, alors que ce procédé de caillage, sous la forme primitive d'addition au lait d'un fragment d'estomac de jeune ruminant, est pratiqué par de nombreux autres peuples nomades, en particulier en Asie.

Un premier type de caillé, l'eedem, est obtenu en ajoutant à du lait chauffé à une température voisine de $80^{\circ} \mathrm{C}$, une quantité suffisante de lait acidifié (tarag, aïrag, etc.) ou de lactosérum acide, afin de provoquer, par l'action combinée de la chute du $\mathrm{pH}$ et de la chaleur, une coagulation rapide des protéines du lait. L'eedem, une fois pressé et égoutté, donne le biaslag, fromage de forme carrée ou rectangulaire, à pâte blanchâtre lisse et assez ferme, dont le goût, légèrement acide, est en général assez fade, surtout lorsque le biaslag est préparé avec du lait écrémé par prélèvement de l'öröm.

Le biaslag peut être consommé frais, peu après sa fabrication. Les éleveurs mongols le font aussi sécher en quelques jours, par temps sec et venteux, sur le toit de leurs yourtes, après l'avoir découpé en tranches. 
Le biaslag séché représente un produit de report de longue conservation, qui est utilisé au fur et à mesure des besoins, le plus souvent réduit en poudre, pour enrichir le thé mongol ou diverses préparations culinaires.

Une variante de la préparation consiste, une fois obtenu l'eedem, à poursuivre la cuisson du caillé pour concentrer le lactosérum et provoquer une caramélisation du lactose. On obtient de cette façon l'eezgij, qui est ensuite séché, conservé et utilisé comme le biaslag.

Un deuxième type de caillé, l'aarts, peut être obtenu de deux façons :

a) Soit à partir du résidu de distillation des laits fermentés acidoalcooliques, qui subsiste dans la chaudière de l'alambic. On récupère cette masse, on l'égoutte. C'est l'arts de distillation.

b) Soit à partir de tarag fraîchement préparé, qui est porté et maintenu à l'ébullition pendant une trentaine de minutes. Le caillé, une fois égoutté, est le targijn aarts dont la qualité organoleptique est, en général, bien supérieure à celle de l'aarts de distillation.

L'aarts peut être consommé frais. En fin de saison, il est surtout utilisé pour préparer des produits de report. Il peut alors être conservé à l'état congelé. Le plus souvent, après avoir été moulé, ou formé à la main ou forcé à travers les mailles d'une passoire pour obtenir de gros filaments, l'aarts ainsi façonné est séché en quelques jours, comme le biaslag, sur le toit des yourtes.

L'aarts dérivant de différents laits fermentés, plus ou moins gras, additionnés ou non de sucre, de diverses baies ou de plantes aromatiques, il en résulte une grande variété de produits secs qui portent chacun une dénomination particulière. L'ensemble est désigné par le terme générique d'aaruul.

\section{获}

Les produits laitiers secs de Mongolie, aaruul, biaslag et eezgij constituent, depuis des siècles, des aliments de base pour les éleveurs nomades mongols. Occupant une place analogue au pain des sédentaires, ces produits laitiers secs sont faciles à transporter, à entreposer et se conservent pendant de longues périodes. On les consomme à tout moment avec des aliments et des plats très variés. En vieillissant, ces produits deviennent excessivement durs et sont alors utilisés, après avoir été émiettés ou réduits en poudre, comme additifs au thé mongol ou à de nombreuses préparations culinaires.

\section{Alcool de lait}

La distillation des divers laits fermentés acido-alcooliques (aïrag, undaa) était autrefois couramment pratiquée en Mongolie, pour 
obtenir un alcool de lait de goût prononcé, le mongol arkihi (litt. alcool mongol). Très apprécié par les Mongols, l'arkhi titrait en moyenne $12^{\circ}$, atteignant parfois $30^{\circ}$ après plusieurs distillations successives. Les alambics utilisés par les éleveurs mongols représentent des appareils de distillation rudimentaires, de conception artisanale, et la distillation des laits fermentés est affaire d'expérience et de doigté. Cette pratique devient d'ailleurs rare, voire réprimée par les autorités.

\section{**}

Trois annexes font suite aux deux articles de Mme Aubin et de MM. Accolas et Deffontaines :

I. Variants génétiques des protéines du lait de vache mongole, par F. Grosclaude.

II. Composition de la matière grasse de laits de vache et de jument mongoles. Premiers résultats, par Simonne Kuzdzal-Savoie et D. Langlois.

III. Etude analytique de la composition des fromages mongols, par R. Jeunet.

Ces différents travaux préliminaires témoignent, à des titres divers, de l'information qu'on peut tirer de l'étude comparative des laits et des produits laitiers provenant de ce lointain pays d'Asie. Il ne fait en effet aucun doute que la Mongolie, pays où sont élevées, dans des conditions de vie assez comparables, la plupart des espèces animales dont le lait est couramment utilisé par les hommes, constitue un terrain privilégié et encore peu exploré, pour les études comparées intéressant la composition des laits. Il est souhaitable, que la coopération scientifique amorcée, dans ce domaine, entre la Mongolie et la France permette de poursuivre activement, dans les années à venir, ce genre d'études.

Reçu pour publication en janvier 1978.

Milk and dairy products in the démocratic Republic of Mongolia. 